\title{
A transfer theorem and stability of Levin-Nohel integro-differential equations
}

Nguyen Tien Dung*

\author{
"Correspondence: \\ dungnt@fpt.edu.vn \\ Department of Mathematics, FPT \\ University, Hoa Lac High Tech Park, \\ Hanoi, Vietnam
}

\begin{abstract}
In this paper we show a connection between Levin-Nohel integro-differential equations and ordinary functional differential equations. Based on this connection, we obtain several new conditions for the stability of the solution, including the famous $3 / 2$ stability criterion.
\end{abstract}

MSC: 34K06; 34K20

Keywords: integro-differential equations; delays; stability; transfer theorem

\section{Introduction}

It is well known that the Levin-Nohel integro-differential equations have many applications in various fields of science and engineering. This class of equations was first studied by Volterra [1] in connection with a biological application. As is well known, the explicit solutions of the Levin-Nohel equations can rarely be obtained. Therefore, it is necessary to investigate stability properties of the solution.

For the Levin-Nohel equations of convolution type with a constant delay, the stability problem was solved by Levin and Nohel $[2,3]$ in the 1960s when they successfully constructed a Liapunov functional. About 15 years ago, a new technique of fixed points developed for studying stability of functional differential equations. Based on this technique, many very nice results have been obtained for asymptotic stability of the non-convolution Levin-Nohel equations with a variable delay (see, for instance, [4-7]).

Let us now consider the non-convolution Levin-Nohel integro-differential equations with several variable delays

$$
\dot{x}(t)+\sum_{k=1}^{N} \int_{t-r_{k}(t)}^{t} a_{k}(t, s) x(s) d s=0, \quad t \geq 0 .
$$

The stability of equation (1) has been recently investigated by the author in $[8,9]$. More specifically, we used the method of fixed points to study the asymptotic stability in [8] and employed a technique of Bohl-Perron type theorems to obtain the sufficient conditions for the exponential stability in [9]. The stability conditions obtained in those two papers are new and improve those established previously by the different authors.

The aim of the present paper is to continue looking for some new stability conditions for (1) and to establish stability conditions for quasilinear Levin-Nohel equations of the

(c) The Author(s) 2017. This article is distributed under the terms of the Creative Commons Attribution 4.0 International License (http://creativecommons.org/licenses/by/4.0/), which permits unrestricted use, distribution, and reproduction in any medium, provided you give appropriate credit to the original author(s) and the source, provide a link to the Creative Commons license, and indicate if changes were made. 
form

$$
\dot{x}(t)+\sum_{k=1}^{N} \int_{t-r_{k}(t)}^{t} a_{k}(t, s) x(s) d s+\sum_{k=N+1}^{M} \int_{t-r_{k}(t)}^{t} a_{k}(t, s) g_{k}(x(s)) d s=0, \quad t \geq 0 .
$$

The method, which will be used in this paper, is very different from that of the papers mentioned above. Our idea is as follows: we first transform Levin-Nohel equations into an ordinary functional differential equation and then we employ known stability conditions for functional differential equations to obtain the stability conditions for Levin-Nohel equations. Since the stability theory of functional differential equations is very rich (see, e.g. [10-12]), we are able to look for a lot of different conditions for the stability of equations (1) and (2).

The rest of this paper is organized as follows. In Section 2, we formulate and prove a transfer theorem for general Levin-Nohel equations. Section 3 is devoted to a study of the stability of linear Levin-Nohel equations with several delays. The stability of quasilinear Levin-Nohel equations is discussed in Section 4. Conclusion is given in Section 5.

\section{Preliminaries and the transfer theorem}

The aim of this section is to provide a transfer theorem for the Levin-Nohel equations with several variable delays of the form

$$
\dot{x}(t)+\sum_{k=1}^{M} \int_{t-r_{k}(t)}^{t} a_{k}(t, s) f_{k}(x(s)) d s=0, \quad t \geq 0,
$$

where, for each $k \in\{1,2, \ldots, M\}, a_{k}(t, s)$ and $r_{k}(t)$ are measurable functions and $f_{k}$ is a Lipschitz function with $f_{k}(0)=0$. We note that the conditions on $f_{k}, k \in\{1,2, \ldots, M\}$ ensure that (3) has the zero solution.

For the convenience of the reader, let us recall some fundamental concepts. For each $t_{0}>0$, we define

$$
m_{k}\left(t_{0}\right)=\inf \left\{t-r_{k}(t): t \geq t_{0}\right\}, \quad m\left(t_{0}\right)=\min \left\{m_{k}\left(t_{0}\right): 1 \leq k \leq M\right\},
$$

and denote by $\mathcal{C}\left(t_{0}\right)$ the space of continuous functions on $\left[m\left(t_{0}\right), t_{0}\right]$ with the supremum norm $\|\cdot\|_{t_{0}}$, which is defined by $\|x\|_{t_{0}}=\sup _{t \in\left[m\left(t_{0}\right), t_{0}\right]}|x(t)|$.

For each $\left(t_{0}, \varphi\right) \in[0, \infty) \times \mathcal{C}\left(t_{0}\right)$, we denote by $x(t)=x\left(t, t_{0}, \varphi\right)$ the unique solution to equation

$$
\dot{x}(t)+\sum_{k=1}^{M} \int_{t-r_{k}(t)}^{t} a_{k}(t, s) f_{k}(x(s)) d s=0, \quad t \geq t_{0},
$$

with the initial data

$$
x(t)=\varphi(t), \quad t \leq t_{0} .
$$

Definition 1 The zero solution of equation (3) is called

(i) stable if for each $\varepsilon>0$ and any $t_{0} \geq 0$ there exists a $\delta>0$ such that $\|\varphi\|_{t_{0}}<\delta$ and $t \geq t_{0}$ imply $\left|x\left(t, t_{0}, \varphi\right)\right|<\varepsilon$. 
(ii) uniformly stable if it is stable and the above $\delta$ is independent of $t_{0}$.

(iii) asymptotically stable if it is stable and $\lim _{t \rightarrow \infty}\left|x\left(t, t_{0}, \varphi\right)\right|=0$.

(iv) uniformly asymptotically stable if it is uniformly stable and $\lim _{t \rightarrow \infty}\left|x\left(t, t_{0}, \varphi\right)\right|=0$.

Definition 2 Equation (3) is called uniformly exponentially stable, if there exist $K>0$, $\lambda>0$ such that any solution of (4), (5) has the estimate

$$
\left|x\left(t, t_{0}, \varphi\right)\right| \leq K\|\varphi(t)\|_{t_{0}} e^{-\lambda\left(t-t_{0}\right)}, \quad t \geq t_{0} .
$$

(We note that $K, \lambda$ do not depend on $t_{0}$.)

Proposition 1 (Mean value theorem for integrals) Let $f$ be a continuous function on $[a, b]$ and $g$ be an integrable function with $g(x) \geq 0($ or $g(x) \leq 0)$ on $[a, b]$. Then there exists a number $c \in[a, b]$ such that

$$
\int_{a}^{b} f(x) g(x) d x=f(c) \int_{a}^{b} g(x) d x .
$$

The main results of this section are stated in the following theorem.

Theorem 1 (Transfer theorem) Let $x(t)$ be the unique solution of equation (3). Suppose there exists a set of indices $I \subset\{1,2, \ldots, M\}$ such that, for each $k \in I$, the kernel $a_{k}(t, s)$ has the following property:

$$
\text { For each } t \geq 0, \int_{t-r_{k}(t)}^{t} a_{k}(t, s) d s \text { exists and } a_{k}(t, s) \geq 0 \text { or } a_{k}(t, s) \leq 0 \quad \forall s .
$$

Then:

1. There exist functions $h_{k}(t), k \in I$ satisfying $0 \leq h_{k}(t) \leq r_{k}(t)$ for all $t \geq 0$ and $x(t)$ is also the solution to the following equation:

$$
\begin{aligned}
& \dot{x}(t)+\sum_{k \in I}\left(\int_{t-r_{k}(t)}^{t} a_{k}(t, s) d s\right) f_{k}\left(x\left(t-h_{k}(t)\right)\right)+\sum_{k \notin I} \int_{t-r_{k}(t)}^{t} a_{k}(t, s) f_{k}(x(s)) d s=0, \\
& \quad t \geq 0 .
\end{aligned}
$$

2. In addition, we assume that, for each $k \in I, f_{k}(x)=x \forall x \in \mathbf{R}$ and

$$
\int_{t-r_{k}(t)}^{t} a_{k}(t, s) d s \geq 0 \quad \forall t \geq 0
$$

Then there exists a function $h(t)$ such that $0 \leq h(t) \leq \max _{k \in I} r_{k}(t)$ for all $t \geq 0$ and $x(t)$ is also the solution to the following equation:

$$
\begin{aligned}
\dot{x}(t) & +\left[\sum_{k \in I}\left(\int_{t-r_{k}(t)}^{t} a_{k}(t, s) d s\right)\right] x(t-h(t))+\sum_{k \notin I} \int_{t-r_{k}(t)}^{t} a_{k}(t, s) f_{k}(x(s)) d s=0, \\
t & \geq 0 .
\end{aligned}
$$




\section{Proof}

1. We first note that equation (3) can be rewritten as follows:

$$
\begin{aligned}
& \dot{x}(t)+\sum_{k \in I} \int_{t-r_{k}(t)}^{t} a_{k}(t, s) f_{k}(x(s)) d s+\sum_{k \notin I} \int_{t-r_{k}(t)}^{t} a_{k}(t, s) f_{k}(x(s)) d s=0, \\
& \quad t \geq 0 .
\end{aligned}
$$

Since $f_{k}, k \in I$ are continuous functions and the kernel $a_{k}(t, s)$ has the property (6), we can apply the mean value theorem for integrals to infer that, for each $k \in I$, there exists $c_{k}(t) \in\left(t-r_{k}(t), t\right)$ such that

$$
\int_{t-r_{k}(t)}^{t} a_{k}(t, s) f_{k}(x(s)) d s=\left(\int_{t-r_{k}(t)}^{t} a_{k}(t, s) d s\right) f_{k}\left(x\left(c_{k}(t)\right)\right) .
$$

Set $h_{k}(t)=t-c_{k}(t)$, then $0 \leq h_{k}(t) \leq r_{k}(t)$ and the above relation becomes

$$
\int_{t-r_{k}(t)}^{t} a_{k}(t, s) f_{k}(x(s)) d s=\left(\int_{t-r_{k}(t)}^{t} a_{k}(t, s) d s\right) f_{k}\left(x\left(t-h_{k}(t)\right)\right), \quad k \in I .
$$

Inserting equalities (11) into (10) leads us to equation (7) and so the proof is complete.

2. Under the assumption $f_{k}(x)=x, k \in I$, equation (7) now reduces to

$$
\begin{aligned}
& \dot{x}(t)+\sum_{k \in I}\left(\int_{t-r_{k}(t)}^{t} a_{k}(t, s) d s\right) x\left(t-h_{k}(t)\right)+\sum_{k \notin I} \int_{t-r_{k}(t)}^{t} a_{k}(t, s) f_{k}(x(s)) d s=0, \\
& \quad t \geq 0 .
\end{aligned}
$$

It follows from the facts $0 \leq h_{k}(t) \leq r_{k}(t), k \in I$, for all $t \geq 0$, that

$t-\max _{k \in I} r_{k}(t) \leq t-h_{k}(t) \leq t$ for all $t \geq 0$. As a consequence, we have

$$
\min _{t-\max _{k \in I} r_{k}(t) \leq u \leq t} x(u) \leq x\left(t-h_{k}(t)\right) \leq \max _{t-\max _{k \in I} r_{k}(t) \leq u \leq t} x(u), \quad t \geq 0 .
$$

This, combined with the condition $\int_{t-r_{k}(t)}^{t} a_{k}(t, s) d s \geq 0, k \in I$, gives us

$$
\sum_{k \in I}\left(\int_{t-r_{k}(t)}^{t} a_{k}(t, s) d s\right) x\left(t-h_{k}(t)\right) \geq\left[\sum_{k \in I}\left(\int_{t-r_{k}(t)}^{t} a_{k}(t, s) d s\right)\right] \min _{t-\max _{k \in I} r_{k}(t) \leq u \leq t} x(u)
$$

and

$$
\sum_{k \in I}\left(\int_{t-r_{k}(t)}^{t} a_{k}(t, s) d s\right) x\left(t-h_{k}(t)\right) \leq\left[\sum_{k \in I}\left(\int_{t-r_{k}(t)}^{t} a_{k}(t, s) d s\right)\right] \max _{t-\max _{k \in I} r_{k}(t) \leq u \leq t} x(u) .
$$

We therefore can obtain the estimate

$$
\begin{aligned}
\min _{t-\max _{k \in I} r_{k}(t) \leq u \leq t} x(u) & \leq \frac{\sum_{k \in I}\left(\int_{t-r_{k}(t)}^{t} a_{k}(t, s) d s\right) x\left(t-h_{k}(t)\right)}{\sum_{k \in I}\left(\int_{t-r_{k}(t)}^{t} a_{k}(t, s) d s\right)} \\
& \leq \max _{t-\max _{k \in I} r_{k}(t) \leq u \leq t} x(u), \quad t \geq 0 .
\end{aligned}
$$


By the intermediate value theorem there exists $c(t) \in\left(t-\max _{k \in I} r_{k}(t), t\right)$ such that

$$
x(c(t))=\frac{\sum_{k \in I}\left(\int_{t-r_{k}(t)}^{t} a_{k}(t, s) d s\right) x\left(t-h_{k}(t)\right)}{\sum_{k \in I}\left(\int_{t-r_{k}(t)}^{t} a_{k}(t, s) d s\right)}, \quad t \geq 0 .
$$

Set $h(t)=t-c(t)$, then $0 \leq h(t) \leq \max _{k \in I} r_{k}(t)$ and the above relation becomes

$$
\begin{aligned}
& \sum_{k \in I}\left(\int_{t-r_{k}(t)}^{t} a_{k}(t, s) d s\right) x\left(t-h_{k}(t)\right) \\
& \quad=\left[\sum_{k \in I}\left(\int_{t-r_{k}(t)}^{t} a_{k}(t, s) d s\right)\right] x(t-h(t)), \quad t \geq 0 .
\end{aligned}
$$

So we can finish the proof by inserting (13) into (12).

Remark 1 If the kernel $a_{k}(t, s)$ and the delays $r_{k}(t), k \in I$, are continuous, then the functions $h_{k}(t), k \in I$, and $h(t)$ are continuous too. This observation will be used to prove Theorems 2,3 , and 5 below.

\section{Linear Levin-Nohel equations}

In this section, we use Theorem 1 to recover some known results and establish new stability conditions for the linear Levin-Nohel equations with several delays of the form (1). We first observe that if $I$ is a subset of $\{1,2, \ldots, N\}$, then the solution $x(t)$ of $(1)$ is also the solution to the equation

$$
\dot{x}(t)+\sum_{k \in I} b_{k}(t) x\left(t-h_{k}(t)\right)+\sum_{k \notin I} \int_{t-r_{k}(t)}^{t} a_{k}(t, s) x(s) d s=0, \quad t \geq 0,
$$

where $b_{k}(t)=\int_{t-r_{k}(t)}^{t} a_{k}(t, s) d s$. Equation (14) is a mixed linear Levin-Nohel equation, its stability has been studied in [8]. Hence, in this whole section, we only consider $I=$ $\{1,2, \ldots, N\}$.

We start with the simplest case, where, for any $k \in I=\{1,2, \ldots, N\}$, the kernel $a_{k}(t, s)$ satisfies the two conditions (6) and (8). Thanks to the second part of Theorem 1 we know that the unique solution $x(t)$ of (1) is also the unique solution to the following equation:

$$
\dot{x}(t)+\left[\sum_{k=1}^{N}\left(\int_{t-r_{k}(t)}^{t} a_{k}(t, s) d s\right)\right] x(t-h(t))=0, \quad t \geq 0,
$$

where the delay $h(t)$ is some function satisfying $0 \leq h(t) \leq \max _{1 \leq k \leq N} r_{k}(t)$ for all $t \geq 0$.

Equation (15) is a functional differential equation with a variable delay of the form

$$
\dot{x}(t)+b(t) x(t-h(t))=0, \quad t \geq 0 .
$$

Obviously, the stability conditions for (16) are well established. Among others we recall here the interesting results from [13-15]. When the delay $h(t)$ is finite we have the famous $3 / 2$ stability criterion. 
Proposition 2 (Myshkis [14] and Yoneyama [15]) Suppose that $b$, $h$ are continuous functions on $\mathbf{R}_{+}, b(t) \geq 0$ for all $t \geq 0$ and $\max _{t \geq 0} h(t)<\infty$.

1. If

$$
\sup _{t \geq 0} \int_{t}^{t+\max _{t \geq 0} h(t)} b(s) d s \leq \frac{3}{2}
$$

then the zero solution of (16) is uniformly stable.

2. If

$$
\liminf _{t \rightarrow 0} b(t)>0, \quad \sup _{t \geq 0} \int_{t}^{t+\max _{t \geq 0} h(t)} b(s) d s<\frac{3}{2}
$$

then the zero solution of (16) is uniformly asymptotically stable.

When the delay $h(t)$ is infinite we have the following results.

Proposition 3 (Graef et al. [13]) Suppose that $b(t) \geq 0$ for all $t \geq 0$ and the delay $h(t)$ is such that

$$
\limsup _{t \rightarrow \infty} \int_{t-h(t)}^{t} b(s) d s<1
$$

Then the zero solution of equation (16) is asymptotically stable if and only if

$$
\int_{0}^{\infty} b(s) d s=\infty
$$

Let us now apply Propositions 2 and 3 to establish the stability conditions for the zero solution of (1).

Theorem 2 Assume that, for any $k \in\{1,2, \ldots, N\}$, the kernel $a_{k}(t, s)$ and the delay $r_{k}(t)$ are continuous functions such that

$$
a_{k}(t, s) \geq 0 \quad \forall(t, s) \quad \text { and } \quad \max _{t \geq 0} r_{k}(t)<\infty
$$

Then the zero solution of (1) is uniformly stable if

$$
\sup _{t \geq 0} \int_{t}^{t+q}\left[\sum_{k=1}^{N}\left(\int_{s-r_{k}(s)}^{s} a_{k}(s, u) d u\right)\right] d s \leq \frac{3}{2},
$$

and it is uniformly asymptotically stable if

$$
\begin{aligned}
& \liminf _{t \rightarrow 0} \sum_{k=1}^{N}\left(\int_{t-r_{k}(t)}^{t} a_{k}(t, s) d s\right)>0, \\
& \sup _{t \geq 0} \int_{t}^{t+q}\left[\sum_{k=1}^{N}\left(\int_{s-r_{k}(s)}^{s} a_{k}(s, u) d u\right)\right] d s<\frac{3}{2},
\end{aligned}
$$

where $q:=\max \left\{\max _{t \geq 0} r_{1}(t), \max _{t \geq 0} r_{2}(t), \ldots, \max _{t \geq 0} r_{N}(t)\right\}$. 
Proof Since $0 \leq h(t) \leq \max _{1 \leq k \leq N} r_{k}(t)$ for all $t \geq 0$, this implies that $\max _{t \geq 0} h(t) \leq q$. Hence, from the non-negative property of $a_{k}(t, s)$ and (17), we can deduce

$$
\sup _{t \geq 0} \int_{t}^{t+\max _{t \geq 0} h(t)}\left[\sum_{k=1}^{N}\left(\int_{s-r_{k}(s)}^{s} a_{k}(s, u) d u\right)\right] d s \leq \frac{3}{2} .
$$

From (19) and the first part of Proposition 2, we can conclude that the zero solution of equation (15) is uniformly stable for any function $h(t)$ satisfying $0 \leq h(t) \leq \max _{1 \leq k \leq N} r_{k}(t)$. So is the zero solution of equation (1) because the solution $x(t)$ of equation (1) is also the solution of equation (15) with some function $h(t)$.

The proof of uniformly asymptotical stability can be done similarity and, hence, we omit it here.

Remark 2 If the kernel $a_{k}(t, s)$ satisfies the two conditions (6) and (8), then $a_{k}(t, s) \geq 0$ because of its continuity. That is why we imposed the condition $a_{k}(t, s) \geq 0 \forall(t, s)$ in Theorem 2.

To provide an illustrative example, let us consider the class of convolution Levin-Nohel equations:

$$
\dot{x}(t)+\int_{t-r}^{t} a(t-s) x(s) d s=0,
$$

where $r>0, a:[0, r] \rightarrow \mathbf{R}_{+}$is continuous. In the view of Theorem 2 , the zero solution of (20) is uniformly asymptotically stable if

$$
\int_{0}^{r} a(s) d s>0 \text { and } r\left(\int_{0}^{r} a(s) d s\right)<\frac{3}{2} .
$$

Meanwhile, Corollary 3.2 in [8] required

$$
\int_{0}^{r} a(s) d s>0 \text { and } \quad \int_{0}^{r} a(s) s d s<1 .
$$

The difference between two conditions confirms that the stability conditions of Theorem 2 are different from that obtained in [8].

Theorem 3 Assume that the kernels $a_{k}(t, s), k \in\{1,2, \ldots, N\}$ are non-negative continuous functions and the delays $r_{k}(t), k \in\{1,2, \ldots, N\}$ are continuous functions such that

$$
\begin{aligned}
& \limsup _{t \rightarrow \infty} \int_{t-\max _{1 \leq k \leq N} r_{k}(t)}^{t}\left[\sum_{k=1}^{N}\left(\int_{s-r_{k}(s)}^{s} a_{k}(s, u) d u\right)\right] d s<1, \\
& \int_{0}^{\infty}\left[\sum_{k=1}^{N}\left(\int_{s-r_{k}(s)}^{s} a_{k}(s, u) d u\right)\right] d s=\infty .
\end{aligned}
$$

Then the zero solution of equation (1) is asymptotically stable.

Proof By using the same arguments presented in the proof of Theorem 2, the desired result follows directly from Proposition 3. 
Remark 3 The results of Theorems 2 and 3 are well known; see e.g. [16]. We restate these results here to illustrate the usefulness of the transfer theorem. By using the transfer theorem, the proofs of Theorems 2 and 3 are very simple.

Let us now consider the case, where, for any $k \in I=\{1,2, \ldots, N\}$, the kernel $a_{k}(t, s)$ fulfils the condition (6). Thanks to the first part of Theorem 1 we know that the unique solution $x(t)$ of (1) is also the unique solution to the following equation:

$$
\dot{x}(t)+\sum_{k=1}^{N}\left(\int_{t-r_{k}(t)}^{t} a_{k}(t, s) d s\right) x\left(t-h_{k}(t)\right)=0, \quad t \geq 0,
$$

which belongs to the class of functional differential equations with several variable delays:

$$
\dot{x}(t)+\sum_{k=1}^{N} b_{k}(t) x\left(t-h_{k}(t)\right)=0, \quad t \geq 0 .
$$

For the stability of (22), we recall the following important result from Theorem 2 in [17].

Proposition 4 (Berezansky and Braverman [17]) Suppose that there exists a set of indices $J \subset\{1,2, \ldots, N\}$ such that

$$
\liminf _{t \rightarrow \infty} \sum_{k \in J} b_{k}(t)>0
$$

and there exists $r(t) \geq 0$ such that for sufficiently large $t$

$$
\int_{t-r(t)}^{t} \sum_{k \in J} b_{k}(s) d s \leq \frac{1}{e}
$$

If

$$
\limsup _{t \rightarrow \infty} \frac{\sum_{k \in J}\left(\left|b_{k}(t) \| \int_{t-h_{k}(t)}^{t-r(t)} \sum_{i=1}^{N}\right| b_{k}(s)|d s|\right)+\sum_{k \notin J}\left|b_{k}(t)\right|}{\sum_{k \in J} b_{k}(t)}<1,
$$

then equation (22) is exponentially stable.

As a product of Proposition 4, we can obtain new conditions for the exponential stability of equation (1) in the next theorem.

Theorem 4 Suppose that there exists a set of indices $J \subset\{1,2, \ldots, N\}$ such that

$$
\liminf _{t \rightarrow \infty} \sum_{k \in J}\left(\int_{t-r_{k}(t)}^{t} a_{k}(t, s) d s\right)>0
$$

and there exists $r(t) \geq 0$ such that for sufficiently large $t$

$$
\int_{t-r(t)}^{t}\left[\sum_{k \in J}\left(\int_{s-r_{k}(s)}^{s} a_{k}(s, u) d u\right)\right] d s \leq \frac{1}{e}
$$




$$
\limsup _{t \rightarrow \infty} \frac{\sum_{k \in J}\left(\left|\int_{t-r_{k}(t)}^{t} a_{k}(t, s) d s\right|\left|\int_{t-r_{k}(t)}^{t-r(t)} \sum_{i=1}^{N}\right| \int_{s-r_{i}(s)}^{s} a_{i}(s, u) d u|d s|\right)+\sum_{k \notin J}\left|\int_{t-r_{k}(t)}^{t} a_{k}(t, s) d s\right|}{\sum_{k \in J} \int_{t-r_{k}(t)}^{t} a_{k}(t, s) d s}<1
$$

then equation (1) is exponentially stable.

Remark 4 If $J=\{j\}$, where $j \in\{1,2, \ldots, N\}$ then the condition (24) reduces to

$$
\limsup _{t \rightarrow \infty}\left(\left|\int_{t-r_{j}(t)}^{t-r(t)} \sum_{i=1}^{N}\right| \int_{s-r_{i}(s)}^{s} a_{i}(s, u) d u|d s|+\frac{\sum_{k \neq j}\left|\int_{t-r_{k}(t)}^{t} a_{k}(t, s) d s\right|}{\int_{t-r_{j}(t)}^{t} a_{j}(t, s) d s}\right)<1 .
$$

If $J=\{1,2, \ldots, N\}$ then the condition (24) reduces to

$$
\limsup _{t \rightarrow \infty} \sum_{k=1}^{N}\left(\frac{\left|\int_{t-r_{k}(t)}^{t} a_{k}(t, s) d s\right|}{\sum_{k=1}^{N} \int_{t-r_{k}(t)}^{t} a_{k}(t, s) d s}\left|\int_{t-r_{k}(t)}^{t-r(t)} \sum_{i=1}^{N}\right| \int_{s-r_{i}(s)}^{s} a_{i}(s, u) d u|d s|\right)<1 .
$$

Remark 5 The exponential stability of equation (1) has been recently discussed in [9]. To the best of our knowledge, that paper seems to be the first one studying the exponential stability of Levin-Nohel integro-differential equations. Theorem 4 thus is an interesting and important contribution because it provides us one more sufficient condition for the exponential stability.

To illustrate Theorem 4 we consider the class of convolution Levin-Nohel equations with two constant delays:

$$
\dot{x}(t)+\int_{t-r_{1}}^{t} a_{1}(t-s) x(s) d s+\int_{t-r_{2}}^{t} a_{2}(t-s) x(s) d s=0,
$$

where $r_{1}, r_{2}>0$ and the kernels $a_{1}:\left[0, r_{1}\right] \rightarrow \mathbf{R}, a_{2}:\left[0, r_{2}\right] \rightarrow \mathbf{R}$ are continuous functions.

Applying Theorem 4 for $J=\{1\},\{2\}$ and $\{1,2\}$ yields the following.

Corollary 1 Suppose at least one of the following conditions holds:

1. $\int_{0}^{r_{1}} a_{1}(s) d s>0$ and there exists $r(t) \geq 0$, such that for sufficiently large $t$

$$
\begin{aligned}
& \left(\int_{0}^{r_{1}} a_{1}(s) d s\right) r(t) \leq \frac{1}{e} \\
& \limsup _{t \rightarrow \infty}\left(\left(\int_{0}^{r_{1}} a_{1}(s) d s+\left|\int_{0}^{r_{2}} a_{2}(s) d s\right|\right)\left|r(t)-r_{1}\right|+\frac{\left|\int_{0}^{r_{2}} a_{2}(s) d s\right|}{\int_{0}^{r_{1}} a_{1}(s) d s}\right)<1 .
\end{aligned}
$$

2. $\int_{0}^{r_{2}} a_{2}(s) d s>0$ and there exists $r(t) \geq 0$ such that for sufficiently large $t$

$$
\begin{aligned}
& \left(\int_{0}^{r_{2}} a_{2}(s) d s\right) r(t) \leq \frac{1}{e} \\
& \limsup _{t \rightarrow \infty}\left(\left(\left|\int_{0}^{r_{1}} a_{1}(s) d s\right|+\int_{0}^{r_{2}} a_{2}(s) d s\right)\left|r(t)-r_{2}\right|+\frac{\left|\int_{0}^{r_{1}} a_{1}(s) d s\right|}{\int_{0}^{r_{2}} a_{2}(s) d s}\right)<1 .
\end{aligned}
$$


3. $\int_{0}^{r_{1}} a_{1}(s) d s+\int_{0}^{r_{2}} a_{2}(s) d s>0$ and there exists $r(t) \geq 0$, such that for sufficiently large $t$

$$
\begin{aligned}
& \left(\int_{0}^{r_{1}} a_{1}(s) d s+\int_{0}^{r_{2}} a_{2}(s) d s\right) r(t) \leq \frac{1}{e}, \\
& \limsup _{t \rightarrow \infty}\left(\left|\int_{0}^{r_{1}} a_{1}(s) d s\right|\left|r(t)-r_{1}\right|+\left|\int_{0}^{r_{2}} a_{2}(s) d s\right|\left|r(t)-r_{2}\right|\right) \\
& \quad<\frac{\int_{0}^{r_{1}} a_{1}(s) d s+\int_{0}^{r_{2}} a_{2}(s) d s}{\left|\int_{0}^{r_{1}} a_{1}(s) d s\right|+\left|\int_{0}^{r_{2}} a_{2}(s) d s\right|} .
\end{aligned}
$$

Then equation (25) is exponentially stable.

\section{Quasilinear Levin-Nohel equations}

The aim of this section is to establish stability conditions for quasilinear Levin-Nohel equations of the form (2). To the best of our knowledge, till now, the stability problem for this new class of the Levin-Nohel equations has not yet been addressed. We impose the assumptions:

(a) For each $k \in\{N+1, \ldots, M\}, g_{k}(0)=0$ and $g_{k}(x)$ is a Lipschitz function, i.e.

$$
\left|g_{k}(x)-g_{k}(y)\right| \leq K_{k}|x-y|, \quad \forall x, y \in \mathbf{R},
$$

where $K_{k}$ is a finite positive constant.

(b) The kernel $a_{k}(t, s)$ is continuous and fulfils the condition (6) for any

$k \in I=\{1,2, \ldots, M\}$.

(c) The delays $r_{k}(t), k \in I$ are continuous with $t-r_{k}(t) \rightarrow \infty$ as $t \rightarrow \infty$.

Under such assumptions, equation (2) with an initial data admits a unique solutions. Moreover, it follows from the first part of Theorem 1 that the solution $x(t)$ of (2) is also the solution to the following quasilinear equation:

$$
\begin{aligned}
\dot{x}(t) & +\sum_{k=1}^{N}\left(\int_{t-r_{k}(t)}^{t} a_{k}(t, s) d s\right) x\left(t-h_{k}(t)\right) \\
& +\sum_{k=N+1}^{M}\left(\int_{t-r_{k}(t)}^{t} a_{k}(t, s) d s\right) g_{k}\left(x\left(t-h_{k}(t)\right)\right)=0, \quad t \geq 0 .
\end{aligned}
$$

This equation belongs to the class of quasilinear functional differential equations with oscillating coefficients of the form

$$
\dot{x}(t)+\sum_{k=1}^{N} b_{k}(t) x\left(t-h_{k}(t)\right)+\sum_{k=N+1}^{M} b_{k}(t) g_{k}\left(x\left(t-h_{k}(t)\right)\right)=0, \quad t \geq 0 .
$$

Our recent paper [18] provides many different conditions for the stability of (27). For the conciseness of the present paper, we only recall here the general results stated in [18] and leave specific cases for the reader. Put

$$
g(t)=\sum_{k=N+1}^{M} K_{k}\left|b_{k}(t)\right|, \quad f(t)=\sum_{k=1}^{N}\left|b_{k}(t)\right|+g(t) .
$$

Theorem 2.1 in [18] reads as follows. 
Proposition 5 (Dung [18]) Suppose that there exists a continuous function $q:[0, \infty) \rightarrow \mathbf{R}$ such that

$$
\begin{aligned}
& \liminf _{t \rightarrow \infty} \int_{0}^{t}\left[\sum_{k=1}^{N} b_{k}(u)+q(u)\right] d u>-\infty, \\
& \sup _{t \geq 0} \int_{0}^{t}\left[|q(s)|+g(s)+\sum_{k=1}^{N}\left(\left|b_{k}(s)\right| \int_{s-h_{k}(s)}^{s} f(u) d u\right)\right] e^{-\int_{s}^{t}\left[\sum_{k=1}^{N} b_{k}(u)+q(u)\right] d u} d s:=\alpha<1 .
\end{aligned}
$$

Then the zero solution of (27) is asymptotically stable if and only if

$$
\int_{0}^{t}\left[\sum_{k=1}^{N} b_{k}(u)+q(u)\right] d u \rightarrow \infty \quad \text { as } t \rightarrow \infty .
$$

Theorem 5 Under the assumptions (a), (b), and (c), the zero solution of equation (2) is asymptotically stable if there exists a continuous function $q:[0, \infty) \rightarrow \mathbf{R}$ such that

$$
\sup _{t \geq 0} \int_{0}^{t}\left[|q(s)|+g(s)+\sum_{k=1}^{N}\left(\left|b_{k}(s)\right| \int_{s-r_{k}(s)}^{s} f(u) d u\right)\right] e^{-\int_{s}^{t}\left[\sum_{k=1}^{N} b_{k}(u)+q(u)\right] d u} d s<1
$$

and

$$
\int_{0}^{\infty}\left[\sum_{k=1}^{N} b_{k}(u)+q(u)\right] d u=\infty
$$

where $b_{k}(t):=\int_{t-r_{k}(t)}^{t} a_{k}(t, s) d s$ and the functions $f, g$ are defined as in (28).

Proof Once again, the proof of this theorem is similar to that of Theorem 2. So we omit it here.

We conclude this section with an example.

Example Consider the equation

$$
\dot{x}(t)+\int_{t-1}^{t} a(b-\sin t) x(s) d s+c \int_{t-|\sin t|}^{t} \frac{\cos (2 s)}{1+t^{2}} \sin (x(s)) d s=0,
$$

where $a>0,0<b<1, c \in \mathbf{R}$.

In view of Theorem 5 , we have

$$
a_{1}(t, s)=a(b-\sin t), \quad a_{2}(t, s)=c \frac{\cos (2 s)}{1+t^{2}} \quad \text { and } \quad g_{2}(x)=\sin (x) .
$$

Hence,

$$
b_{1}(t)=a(b-\sin t), \quad b_{2}(t)=c \frac{\sin (2 t)-\sin (2 t-2|\sin t|)}{2}
$$

and

$$
g(t)=\left|c \frac{\sin (2 t)-\sin (2 t-2|\sin t|)}{2\left(1+t^{2}\right)}\right| \leq|c|, \quad f(t)=\left|b_{1}(t)\right|+g(t) \leq a b+a+|c| .
$$


Since $\int_{0}^{t} b_{1}(s) d s=a b t-a(1-\cos t) \geq a b t-2 a$, the condition (30) is satisfied with $q(t)=0$. Consequently, the zero solution of (31) is asymptotically stable if

$$
\sup _{t \geq 0} \int_{0}^{t}\left[|c|+\left((a b+a) \int_{s-1}^{s}(a b+a+|c|) d u\right)\right] e^{-a b(t-s)-a(\cos t-\cos s)} d s<1 .
$$

It follows from the fact that $\int_{0}^{t} e^{-a b(t-s)-a(\cos t-\cos s)} d s \leq e^{2 a} \int_{0}^{t} e^{-a b(t-s)} d s \leq \frac{e^{2 a}}{a b}, \forall t \geq 0$, that (32) holds true if

$$
e^{2 a}\left(\frac{|c|}{a b}+\frac{(a b+a+|c|)(b+1)}{b}\right)<1,
$$

which is satisfied with $a=0.1, b=0.9$ and $c= \pm 0.03$.

\section{Conclusion}

In this paper, we proved a transfer theorem for the Levin-Nohel equations. Because of its simplicity, the transfer theorem provides us with an effective method to investigate the stability of the Levin-Nohel equations. Each of the known stability conditions for the corresponding functional differential equations may give us a stability condition for the original Levin-Nohel equations.

By using the transfer theorem, we obtain simple proofs for some well-known results (Theorems 2 and 3) and new stability conditions (Theorems 4 and 5) for the Levin-Nohel equations.

\section{Competing interests}

The author declares that he has no competing interests.

\section{Author's contributions}

All author has written this paper. The author has read and approved the final version of the manuscript.

\section{Acknowledgements}

The author would like to thank the anonymous referees for their valuable comments and for the reference to $[14,16]$.

Received: 30 October 2016 Accepted: 23 February 2017 Published online: 01 March 2017

\section{References}

1. Volterra, V: Sur la théorie mathématique des phénomès héréditaires. J. Math. Pures Appl. 7, 249-298 (1928)

2. Levin, JJ, Nohel, JA: On a system of integrodifferential equations occurring in reactor dynamics. J. Math. Mech. 9, 347-368 (1960)

3. Levin, JJ, Nohel, JA: On a nonlinear delay equation. J. Math. Anal. Appl. 8, 31-44 (1964)

4. Burton, TA: Fixed points and stability of a nonconvolution equation. Proc. Am. Math. Soc. 132, 3679-3687 (2004)

5. Burton, TA: Fixed points, stability, and harmless perturbations. Fixed Point Theory Appl. 1, 35-46 (2005)

6. Burton, TA: The case for stability by fixed point theory. Dyn. Contin. Discrete Impuls. Syst. Ser. A Math. Anal. 13B(suppl), 253-263 (2006)

7. Becker, LC, Burton, TA: Stability, fixed points and inverse of delays. Proc. R. Soc. Edinb. 136A, 245-275 (2006)

8. Dung, NT: New stability conditions for mixed linear Levin-Nohel integro-differential equations. J. Math. Phys. 54, 082705 (2013)

9. Dung, NT: On exponential stability of linear Levin-Nohel integro-differential equations. J. Math. Phys. 56, 022702 (2015)

10. Halanay, A: Differential Equations: Stability, Oscillations, Time Lags. Academic Press, New York (1966)

11. Hale, IK, Verduyn Lunel, SM: Introduction to Functional-Differential Equations. Applied Mathematical Sciences, vol. 99. Springer, New York (1993)

12. Krisztin, T: On stability properties for one-dimensional functional-differential equations. Funkc. Ekvacioj 34(2), 241-256 (1991)

13. Graef, JR, Qian, C, Zhang, B: Asymptotic behavior of solutions of differential equations with variable delays. Proc. Lond. Math. Soc. 81, 72-92 (2000)

14. Myshkis, AD: Linear Differential Equations with Retarded Argument. Nauka, Moscow (1972) (in Russian)

15. Yoneyama, T: On the $3 / 2$ stability theorem for one-dimensional delay-differential equations. J. Murh. Anal. Appl. 125, 161-173 (1987) 
16. Gusarenko, SA, Domoshnitskiǐ, Al: Asymptotic and oscillation properties of first-order linear scalar functional-differential equations. Differ. Equ. 25(12), 1480-1491 (1989)

17. Berezansky, L, Braverman, E: Explicit exponential stability conditions for linear differential equations with several delays. J. Math. Anal. Appl. 332(1), 246-264 (2007)

18. Dung, NT: An asymptotic stability theorem for quasilinear delay differential equations with oscillating coefficients. Int. J. Math. 24(11), 1350092 (2013) 14 pp

Submit your manuscript to a SpringerOpen ${ }^{\circ}$ journal and benefit from:

- Convenient online submission

Rigorous peer review

- Immediate publication on acceptance

Open access: articles freely available online

- High visibility within the field

- Retaining the copyright to your article 